\title{
Once-daily fluticasone furoate/vilanterol versus twice daily combination therapies in asthma-mixed treatment comparisons of clinical efficacy
}

Henrik Svedsater ${ }^{1 *}$, Gillian Stynes ${ }^{1}$, Jaro Wex ${ }^{1}$, Lucy Frith ${ }^{2}$, David Leather ${ }^{2}$, Emanuela Castelnuovo ${ }^{3}$, Michelle Detry ${ }^{4}$ and Scott Berry ${ }^{4}$

\begin{abstract}
Background: Fluticasone furoate (FF)/vilanterol (VI) is a once-daily inhaled corticosteroid (ICS)/long-acting beta 2 agonist (LABA) combination. FFNI, 92/22mcg and 184/22mcg, are approved in Europe as maintenance therapy in persistent asthma. We report data from mixed treatment comparisons (MTC) of once-daily FFNI against established twice-daily ICS/LABA combination therapies on clinical efficacy outcomes.

Methods: Data from 31 parallel-group randomised controlled trials (RCTs) of ICS/LABA, of $\geq 8$ weeks' duration in patients aged $\geq 12$ years with asthma, identified by systematic review, were analysed using covariate-adjusted Bayesian hierarchical models for four efficacy outcomes (primary analysis). Lung function, assessed by change from baseline morning peak expiratory flow (PEF) ( $n=18$ studies) and forced expiratory volume in $1 \mathrm{~s}\left(\mathrm{FEV}_{1}\right)(n=28)$, was the outcome of primary interest. Secondary objectives were assessment of relative efficacy in terms of exacerbation rates $(n=6)$ and health status $(n=7)$. Overall, 24 different treatment arms were included in the MTC; we report findings comparing FF/NI (92/22mcg and 184/22mcg) with fluticasone propionate/salmeterol (FP/SAL) (250/50mcg and 500/ $50 \mathrm{mcg}$ ) and budesonide/formoterol (BUD/FORM) (320/9mcg and 640/18mcg).

Results: For PEF (margin = $12 \mathrm{l} / \mathrm{min}$ ), FFNI 92/22mcg demonstrated $\geq 94 \%$ probability and FFNI 184/22mcg $>99 \%$ probability of non-inferiority to corresponding doses of both FP/SAL and BUD/FORM. For FEV $($ margin $=100 \mathrm{ml}$ ), FFMI demonstrated $\geq 98 \%(92 / 22 \mathrm{mcg})$ and $>99 \%(184 / 22 \mathrm{mcg})$ probability of non-inferiority to both FP/SAL and BUD/FORM. Findings for exacerbations were inconclusive due to lack of data: FF/VI 92/22mcg demonstrated $74 \%$ and $82 \%$ probability of non-inferiority (margin $=10 \%$ ) to FP/SAL 250/50mcg and BUD/FORM 320/9mcg, respectively. For Asthma Quality of Life Questionnaire (AQLQ) score, FF/NI 92/22mcg demonstrated $>99 \%$ and $90 \%$ probability of non-inferiority (margin $=0.25$ ) to FP/SAL 250/50 mcg and BUD/FORM 320/9mcg. Data were unavailable to assess non-inferiority of FF/VI 184/22mcg on exacerbations or AQLQ.

Conclusions: Both strengths of once-daily FFNI in asthma were comparable with corresponding doses of twice-daily FP/SAL and BUD/FORM in terms of lung function in this MTC analysis. FF/ 92/22mcg was comparable with FP/SAL and BUD/FORM on AQLQ, but exacerbation results were inconclusive. Model limitations include disconnected treatment networks and variability across studies. Our data support previous RCT findings suggesting that the efficacy of once-daily FFNI in improving lung function and health status in asthma is comparable with twice-daily ICS/LABAs.
\end{abstract}

Keywords: Asthma, Fluticasone furoate, ICS/LABA, Mixed treatment comparison, Network meta-analysis, Vilanterol

\footnotetext{
* Correspondence: henrik.x.svedsater@gsk.com

${ }^{1}$ Value Evidence and Outcomes, GlaxoSmithKline, Stockley Park West, Blg 10,

1-3 Iron Bridge Road, Uxbridge, Middlesex UB11 1BT, UK

Full list of author information is available at the end of the article
} 


\section{Background}

Fluticasone furoate (FF)/vilanterol (VI) is an inhaled corticosteroid (ICS)/long-acting beta 2 agonist (LABA) combination approved in 2013 in Europe and Japan for the treatment of asthma. Both FF and VI have been shown to display 24-h activity in pre-clinical studies [1, 2], and a single daily dose of FF/VI has been shown to produce long-lasting improvement in lung function in patients with asthma [3]. Once-daily FF/VI, at strengths of $92 / 22$ mcg and 184/22 mcg, has been shown to be well tolerated, with no safety profile findings of significant clinical concern, in a 12-month randomised controlled trial in patients aged $\geq 12$ years with persistent moderate-severe asthma [4]. FF/VI therefore represents the first once-daily combination therapy approved for use in asthma.

The efficacy of FF/VI in asthma has been assessed in several randomised controlled trials (RCTs). The effects of once daily FF/VI 92/22 mcg (delivered dose; nominal dose is $100 / 25 \mathrm{mcg}$ ) on lung function and patient-reported health status have been compared in RCTs with FF 100 mcg [3, 5], and once-daily FF/VI 184/22 mcg (delivered dose; nominal dose is $200 / 25 \mathrm{mcg}$ ) has been compared with once-daily FF 200 mcg alone and twice-daily FP 500 mcg [6]. Once-daily FF/VI 92/22 mcg has also been compared in a head-to-head study with twice daily FP/SAL $250 / 50 \mathrm{mcg}$ [7]. One study of the efficacy in reducing exacerbation rate of FF/VI 92/22 mcg compared with FF monotherapy has been reported [5]. However, the efficacy of once-daily FF/VI has not, as of the time of writing, been directly compared in any RCT with that of twice-daily combination therapies other than FP/SAL; some of which, such as budesonide (BUD)/formoterol (FORM), are widely used in the clinical management of asthma.

We sought to evaluate the relative therapeutic efficacy of FF/VI in asthma by making maximal use of the body of RCT data available. Indirect treatment comparisons, such as the mixed treatment comparison (MTC), provide a means of estimating the relative efficacy of treatments that have not been directly compared in an RCT, and broaden the evidence base for those treatments which have already been compared in head-to-head studies. We applied the MTC approach to compare the treatment efficacy of oncedaily FF/VI 92/22 mcg and 184/22 mcg with corresponding strengths of alternative licensed twice-daily ICS/LABA combination therapies on four clinically-relevant outcomes. For each outcome, we combined data from separate RCTs, all of which were identified through a systematic literature review and involved at least one ICS/LABA comparator, in Bayesian, hierarchical models. These models were used to make inferences about relative treatment efficacy within the combined ICS/LABA therapy class and derive probabilities of non-inferiority and superiority for four clinically relevant outcomes: peak expiratory flow (PEF), forced expiratory volume in $1 \mathrm{~s}\left(\mathrm{FEV}_{1}\right)$, moderate/ severe exacerbation rate, and asthma quality of life questionnaire (AQLQ/AQLQ(S) Total score). The primary focus of the analyses was on the non-inferiority results.

\section{Methods}

\section{Systematic literature review}

A systematic literature review was conducted to identify Phase III and Phase IV parallel-group RCTs of any ICS/LABA maintenance therapies vs any drug, placebo or standard of care comparator, of $>8$ weeks duration, in patients aged $\geq 12$ years with asthma who were receiving ICS or ICS/LABA maintenance therapy at randomisation. Studies that required patients to be uncontrolled/symptomatic at baseline were eligible for inclusion in the MTC. Studies that included patients who were controlled/asymptomatic at baseline, predominantly recruited stable patients (explicitly defined or suggested by baseline averages of key parameters) or required patients to have a history of stable asthma, or did not report control or symptom status at randomisation were excluded. Additionally, studies containing $\geq 1$ flexible dosing or dose-ranging arm were excluded.

A secondary MTC analysis, the 'ICS-only subset analysis', was conducted including only the subset of studies requiring patients to be treated with ICS only at baseline, i.e. LABA use was not permitted at randomisation. This approach identified studies in patient populations likely to be eligible for treatment with FF/VI in accordance with its European licence.

FF/VI trials were identified internally, and additional studies were identified through the systematic searching of clinical publication databases and clinical trial registers. Additionally, references in retrieved articles and relevant systematic reviews were checked for further studies that might fulfil the inclusion criteria. No date limits were applied to the searches.

\section{Outcome assessment}

The outcomes assessed were change from baseline in lung function outcomes: PEF and $\mathrm{FEV}_{1}$, annual rate of moderate/severe exacerbations, and change from baseline in AQLQ health status questionnaire Total score. Moderate/severe exacerbations were defined according to the American Thoracic Society/European Respiratory Society Task Force recommendation [8] as deteriorations of asthma requiring the use of systemic/oral corticosteroids for $\geq 3$ days, or emergency department visit or in-patient hospitalisation due to asthma requiring the use of systemic corticosteroids.

The outcome of primary interest, effect of treatment on lung function, was studied through the assessment of two measures of airflow limitation: PEF and FEV ${ }_{1}$. These measures are typically well correlated with each other [9]. 
For each outcome, studies identified through the systematic literature review were included in the MTC if they reported the precise outcome or sufficient calculable information in a suitable format. For PEF, to minimise study-to-study variability, studies were included only if they reported mean changes from baseline averaged over the entire duration of the trial. For exacerbations, studies were included in the MTC if they reported either the rate or number of moderate/severe exacerbations as defined above. Studies that allowed changes in symptoms, rescue use or lung function to be classed as exacerbations were excluded, as were those that withdrew patients once they experienced an exacerbation. With a single exception of arms consisting of steroid-naive patients in one study [10], all treatment arms from included studies were incorporated into the MTC.

The selection of margins that were used in the probabilistic assessment of non-inferiority for each outcome was informed by the margins specified in the design of previously reported comparative studies. For PEF, non-inferiority margins of $12 \mathrm{l} / \mathrm{min}$ and $15 \mathrm{l} / \mathrm{min}$ were selected on the basis of published guidelines [11]. For exacerbations, event rate ratios of 0.1 and 0.2 were used as non-inferiority margins on the basis of previous comparative studies [12]. For $\mathrm{FEV}_{1}$ (75 ml, $100 \mathrm{ml}$ and $125 \mathrm{ml}$ ) and AQLQ (0.25 and 0.5), the non-inferiority margins used were based upon the accepted minimal clinically important difference (MCID) $[13,14]$.

\section{Modelling strategy}

The MTC modelling approach used in this analysis employed a Bayesian, hierarchical methodology to estimate relative treatment effects, accounting for variability across studies by parameterising the study effect. The Bayesian approach [15-17] was decided upon prior to the commencement of the systematic literature review. This methodology enables the comparison of the effects of treatments that have never been directly compared in the same clinical trial, and is compatible with inference from weak or disconnected treatment networks [18].

For each outcome, a hierarchical random effects model was constructed in which the effect of each included study $\alpha$ was modelled with a distribution $\alpha_{S} \sim N\left(\mu, \tau^{2}\right)$. The two parameters $\mu$ and $\tau$ were then modelled with second-level hyperpriors and a posterior distribution created. Treatment effects, with independent prior distributions, were modelled separately as a single parameter, thus enabling probabilistic treatment comparisons to be derived together with credible intervals $(\mathrm{CrI})$ for the differences in effect sizes.

For PEF, FEV 1 and AQLQ Total score, the continuous change from baseline outcomes was modelled using Normal distributions. The mean treatment effect was modelled with the following distribution: $Y \sim N\left(\alpha_{s}+\theta_{t}+\beta Z, \sigma^{2}\right)$, with the non-informative prior $\sigma_{\alpha}^{2} \sim$ Inverse-Gamma(0.001,
$0.001)$ and hyperpriors $\mu_{s} \sim N\left(0,10^{2}\right)$ and $\tau^{2} \sim$ Inverse $\operatorname{Gamma}(0.001,0.001)$. The parameters $\alpha$ and $\theta$ represent, respectively, the studies included in the analysis, and the treatment regimen effects. The $Z$ 's represent the covariates and the $\beta$ 's represent the coefficients (i.e. the covariate effects). Each treatment effect is modelled independently with the flat prior distribution $N\left(0,100^{2}\right)$.

For exacerbations, the yearly rate was modelled using a Poisson distribution, as follows: Exac $\sim$ Poisson(Rate son-years), in which $\log ($ Rate $)=\alpha_{s}+\theta_{t}+\beta Z$. Priors and model parameters for the study and treatment effects were defined as for the other three outcomes; treatment regimen effects are represented by $\theta$. Each treatment effect is modelled independently with the flat prior distribution $N\left(0,5^{2}\right)$. The placebo arms are designated as $t=0$ and are used as the baseline treatment. The original analysis plan was to use a standard deviation of 100 for the treatment effects. This represents a very flat, non-informative prior distribution. However, the outcome of one trial arm with 0 events makes for a non-identifiable outcome which, combined with the very flat prior, creates numerical stability issues. Therefore, a vague prior of 5 is selected for the treatment arms, creating stable estimates and more interpretable model results.

Rate was defined as number of exacerbations divided by person-years of follow-up. Person-years of follow-up were computed directly if both the rate and number of events were available or were estimated if neither were available. When estimated, subjects not lost to follow-up were assumed to have had complete (100\%) follow-up. Subjects lost to follow-up were assumed to have $50 \%$ of the possible follow-up. When the number of events was not reported, the rate and estimated person-years of follow-up were used to estimate the number of moderate/severe exacerbation events.

The following covariates were included in the full covariate model for all outcomes: study duration; age at baseline; gender; baseline mean $\mathrm{FEV}_{1}$. The continuous covariatesage, gender and baseline mean $\mathrm{FEV}_{1}$-were normalised. Specifically, age was normalised by subtracting 40 years of age; the resulting covariate is "Age-40". Gender is represented as the percentage of males in a treatment arm. Gender was normalised by subtracting $40 \%$ from the treatment arm population of males, thus the covariate is "\%Male-40". Baseline $\mathrm{FEV}_{1}$ is normalised by subtracting 2.4 ; the resulting covariate is " $\mathrm{FEV}_{1}-2.4$ ". The single categorical variable, length of study, had a designated reference group of studies 40-60 weeks in length, i.e. those that were approximately 1 year in length.

In treatment arms with missing covariate information, mean values were imputed across all treatment arms. Attempts were made to fit full covariate models for all outcomes of interest, but this could not be achieved for exacerbations as data limitations meant the full covariate 
model did not converge. As such, exacerbation data are reported only for the time-covariate model, in which only study duration was modelled as a covariate. For the other three outcomes, findings from the full covariate model are reported.

Model fit was assessed by evaluating the difference between the model-estimated values and observed values, divided by the estimated standard deviation (SD).

All analyses were carried out using standard Markov Chain Monte Carlo methodology, utilising adaptive Metropolis-Hastings steps where applicable [19], and were performed using custom software written in ANSIstandard Fortran (Berry Consultants LLC, Austin, TX). The software used was independently validated with duplicate code written in R ( $\mathrm{R}$ Development Core Team, Vienna, Austria).

\section{Sensitivity analysis}

Sensitivity analyses, in which studies that were excluded under the primary analysis criteria were added to the model, were performed for the PEF and exacerbation rates outcomes of interest, using the same modelling approach as in the main analyses. For the outcome of change from baseline PEF, the sensitivity analysis included an additional nine studies that were excluded from the main analysis because they did not report change in PEF from baseline to study end. The PEF sensitivity analysis network was thus constructed using data from 27 studies. For exacerbations, one of the sensitivity analyses included an additional six studies that were excluded from the main analysis because they withdrew patients and discontinued their follow-up after they reported an exacerbation, for a sensitivity analysis network consisting of data from 12 studies.

In separate sensitivity analyses performed for the exacerbation rate network, the data for the original six studies were re-analysed using alternate versions of the personyears calculation for those cases where person-years were estimated. Two such analyses were conducted, using an assumed follow-up time for patients lost to follow-up of $25 \%$ or $75 \%$, respectively, rather than $50 \%$ as in the main analysis.

\section{Assessment of alternative modelling approaches}

Two post-hoc validation analyses were performed on the same dataset using alternative methods in order to evaluate the extent to which outcomes were susceptible to the primary model chosen: a frequentist analysis using a random effects model with fixed study and treatment effects, reporting $p$-values was performed on all four outcomes of interest using R (lme4 package); and a pairwise contrast analysis using a Bayesian random effects model was performed on the $\mathrm{FEV}_{1}$ data only using geMTC software [20] running WinBUGS [21]. As geMTC did not enable automated analyses of rates with Poisson distributions, exacerbation rates were approximated as continuous variables, comparing rate differences rather than rate ratios. In the validation analyses, point estimates with confidence or credible intervals were calculated, with the frequentist analyses also reporting $p$-values.

\section{Results \\ Study selection}

Thirty-six unique studies were considered for inclusion in the MTC. A total of 31 parallel-group trials, with 23 different drug treatments plus placebo, were included in at least one of the four primary analyses (Fig. 1). For the primary analysis populations, eighteen studies were included in the analysis of change from baseline PEF; 28 for $\mathrm{FEV}_{1}$; seven for $\mathrm{AQLQ}$; and six for exacerbation rates (Additional file 1: e-table 1).

For the ICS-only subset analysis, the study sets were the same for PEF and exacerbations, and slightly smaller for $\mathrm{FEV}_{1}$ and AQLQ (26 and six studies, respectively). The studies and treatment arms included in the MTC are summarised in Table 1. Reasons for exclusion of studies from each analysis are outlined in the Additional file 1. The networks of studies included for each outcome of interest are shown in Fig. 1 and Additional file 1: Supplementary Figure 1.

\section{Primary analysis \\ Change from baseline PEF}

Placebo treatment was associated with a slight mean (SD) decrease from baseline of -1.7 (8.9) $\mathrm{l} / \mathrm{min}$. All ICS/ LABA combination therapies included in the model were associated with mean (SD) improvements from baseline PEF ranging from 19.65 (8.55) l/min with BUD/ FORM 100/6 mcg to 49.94 (7.63) $1 / \mathrm{min}$ with FF/VI 184/ $22 \mathrm{mcg}$ (Fig. 2 and Additional file 1: e-table 2).

Based on a non-inferiority margin of $12 \mathrm{l} / \mathrm{min}, \mathrm{FF} / \mathrm{VI}$ 92/ 22 mcg demonstrated $97 \%$ and $94 \%$ probability of noninferiority to corresponding doses of twice-daily FP/SAL 250/50 mcg and BUD/FORM 320/9 mcg, respectively (Table 2). On the same margin, FF/VI 184/22 mcg demonstrated $>99 \%$ probability of non-inferiority to corresponding doses of both FP/SAL 500/50 mcg and BUD/FORM 640/18 mcg. Based on a non-inferiority margin of $15 \mathrm{l} / \mathrm{min}$, FF/VI 92/22 mcg demonstrated $99 \%$ and $98 \%$ probability of non-inferiority to corresponding doses of twice-daily FP/SAL $250 / 50 \mathrm{mcg}$ and BUD/FORM $320 / 9 \mathrm{mcg}$, respectively. On the same margin, FF/VI 184/22 mcg demonstrated >99\% probability of non-inferiority to doses of both FP/SAL $500 / 50 \mathrm{mcg}$ and BUD/FORM 640/18 mcg. Of the covariates analysed, only baseline $\mathrm{FEV}_{1}$ had a significant effect on PEF, though the credible interval was wide (Additional file 1: e-table 3). 


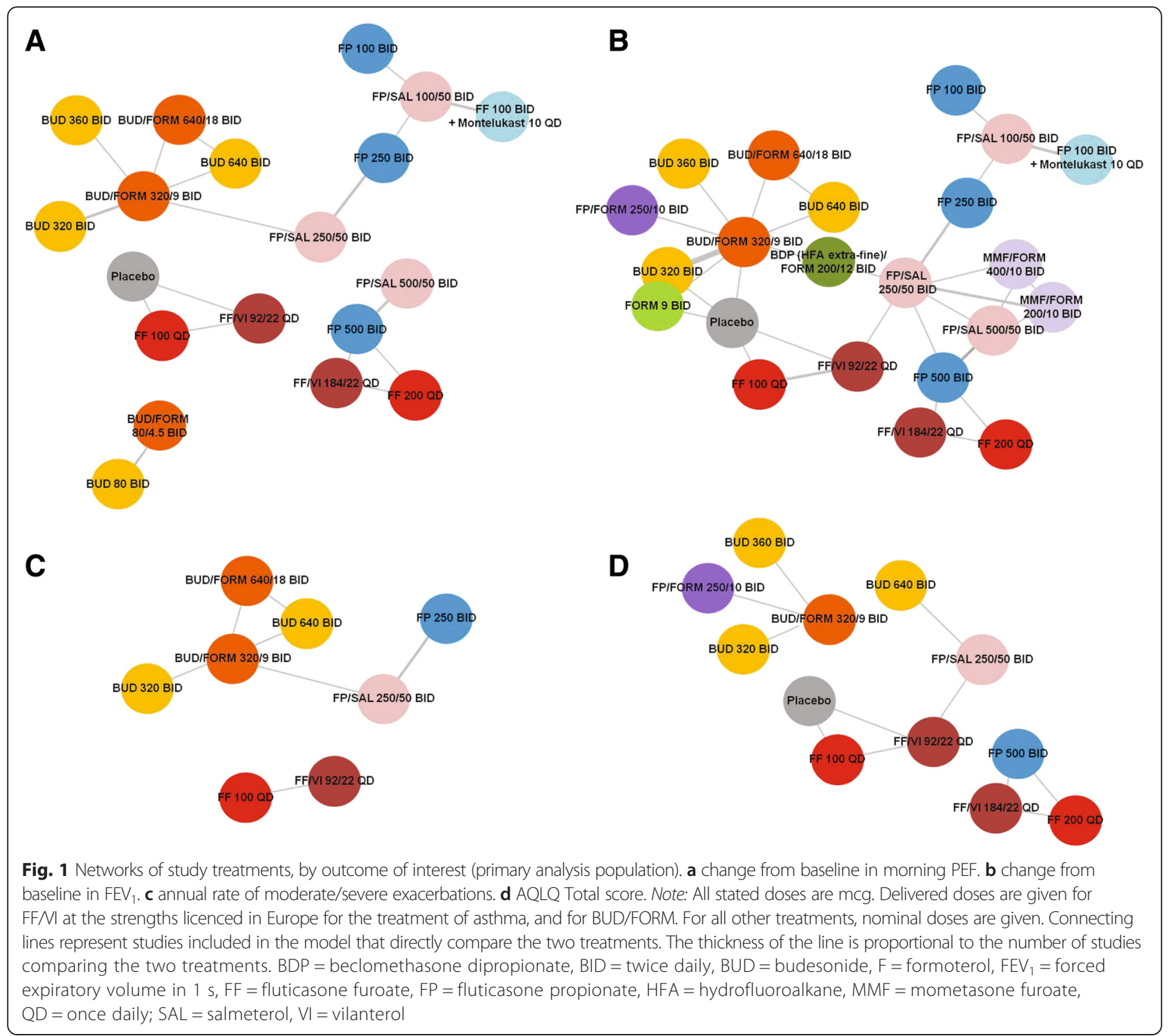

\section{Change from baseline FEV}

All ICS/LABA combination therapies were associated with estimated improvements from baseline $\mathrm{FEV}_{1}$ of $\geq 147 \mathrm{ml}$, whereas treatment with placebo was associated with a mean (SD) decrease from baseline of 32 (58) $\mathrm{ml}$. ICS/LABA treatment was associated with mean (SD) improvements from baseline, ranging from 147 (54) ml with FP/SAL 100/ $50 \mathrm{mcg}$ to 353 (67) $\mathrm{ml}$ with FF/VI 184/22 mcg (Fig. 2 and Additional file 1: e-table 2).

Based on the most stringent of the three non-inferiority margins assessed, $75 \mathrm{ml}$, FF/VI 92/22 mcg demonstrated $92 \%$ and $91 \%$ probability of non-inferiority to corresponding doses of FP/SAL 250/50 mcg and BUD/FORM $320 / 9$ mcg, respectively. On the same margin, FF/VI 184/ $22 \mathrm{mcg}$ demonstrated $>99 \%$ probability of non-inferiority to corresponding doses of $\mathrm{FP} / \mathrm{SAL} 500 / 50 \mathrm{mcg}$ and BUD/FORM 640/18 mcg (Table 2). On a slightly less stringent margin of $100 \mathrm{ml}$, representing just under half of the MCID [13], FF/VI 92/22 mcg demonstrated $99 \%$ and $98 \%$ probability, respectively, of noninferiority to corresponding doses of FP/SAL 250/50 mcg and BUD/FORM 320/9 mcg. On the same margin, FF/VI 184/22 mcg demonstrated $>99 \%$ probability of non-inferiority to corresponding doses of FP/SAL 500/ $50 \mathrm{mcg}$ and BUD/FORM 640/18 mcg. Based on the non-inferiority margin of $125 \mathrm{ml}, \mathrm{FF} / \mathrm{VI}$ 92/22 mcg and $184 / 22$ mcg demonstrated $>99 \%$ probability of noninferiority to corresponding doses of FP/SAL 250/50 $\mathrm{mcg}$ and 500/50 $\mathrm{mcg}$ and BUD/FORM 320/9 mcg and $640 / 18 \mathrm{mcg}$, respectively. None of the model covariates 
Table 1 Summary of studies and treatment arms included in the primary mixed treatment comparison analysis

\begin{tabular}{|c|c|c|c|}
\hline \multirow[b]{2}{*}{ Total studies } & \multicolumn{2}{|l|}{ N (\%) } & \multirow{2}{*}{$\begin{array}{l}N(\%) \\
75\end{array}$} \\
\hline & 31 & Total treatment arms & \\
\hline Endpoint reported & & Placebo & $2(3)$ \\
\hline $\begin{array}{l}\text { Change from baseline } \\
\text { in FEV }\end{array}$ & $28(90)$ & FF/NI 92/22 QD & $3(4)$ \\
\hline $\begin{array}{l}\text { Change from baseline } \\
\text { in PEF }\end{array}$ & $18(58)$ & FF/VI 184/22 QD & $1(1)$ \\
\hline Annual rate of exacerbations & $6(19)$ & FF 100 QD & $2(3)$ \\
\hline $\begin{array}{l}\text { Change from baseline } \\
\text { in AQLQ }\end{array}$ & $7(23)$ & FF 200 QD & $1(1)$ \\
\hline Mean age reported & 41.74 & FP/SAL 100/50 BID & $8(11)$ \\
\hline Mean percent male & 40.51 & FP/SAL 250/50 BID & $11(15)$ \\
\hline \multirow[t]{17}{*}{ Mean baseline $\mathrm{FEV}_{1}$} & 2.30 & FP/SAL 500/50 BID & $5(7)$ \\
\hline & & BUD/FORM 320/9 BID & $12(16)$ \\
\hline & & BUD/FORM 640/18 BID & $1(1)$ \\
\hline & & BUD/FORM 80/4.5 BID & $2(3)$ \\
\hline & & BUD 360 BID & $5(7)$ \\
\hline & & BUD 640 BID & $2(3)$ \\
\hline & & BUD 360 BID & $1(1)$ \\
\hline & & BUD 80 BID & $1(1)$ \\
\hline & & $\begin{array}{l}\text { BDP (HFA extra-fine)/ } \\
\text { FORM 200/12 BID }\end{array}$ & $2(3)$ \\
\hline & & FORM 9 BID & $1(1)$ \\
\hline & & FP $250 \mathrm{BID}$ & $3(4)$ \\
\hline & & FP 500 BID & $4(5)$ \\
\hline & & FP $100 \mathrm{BID}$ & $1(1)$ \\
\hline & & $\begin{array}{l}\text { FP } 100 \text { BID + } \\
\text { Montelukast } 10 \text { QD }\end{array}$ & $3(4)$ \\
\hline & & FP/FORM 250/10 BID & $1(1)$ \\
\hline & & MMF/F 200/10 BID & $2(3)$ \\
\hline & & MMF/F 400/10 BID & $1(1)$ \\
\hline
\end{tabular}

Note: All stated doses are mcg. Delivered doses are given for FF/ $\mathrm{NI}$ at the strengths licenced in Europe for the treatment of asthma, and for BUD/FORM. For all other treatments, nominal doses are given

$A Q L Q$ Asthma quality of life questionnaire, $B D P$ Beclomethasone dipropionate, $B I D$ Twice daily, BUD Budesonide, FORM Formoterol, $F E V_{1}$ Forced expiratory volume in one $s$, FF Fluticasone furoate, FP Fluticasone propionate, HFA Hydrofluoroalkane, MMF Mometasone furoate, PEF Peak expiratory flow, $Q D$ once daily, SAL Salmeterol, TIO Tiotropium, VI Vilanterol

were found to have a statistically significant effect on outcomes (Additional file 1: e-table 3).

\section{Annual moderate/severe exacerbation rate}

Relative to a benchmark rate of 1 , based upon data from the placebo arms of studies included in the model, ICS/ LABA combination therapies were associated with estimated standardised event rate ratios of $\leq 0.203$. The CrI for the reduction in event rate ratio for all active treatments excluded 1 . The greatest reduction in event rate ratio relative to placebo, 0.168 (95\% CrI: 0.088-0.236), was seen with twice-daily FP/SAL 250/50 mcg. The estimated event rate ratio for once-daily FF/VI 92/22 mcg was 0.174 (95 \% CrI: 0.070-0.443) (Fig. 2 and Additional file 1: e-table 2).

Because of the disconnected network for the exacerbation rate endpoint, the model included only one covariate, study duration, as the addition of further covariates resulted in the failure of the model to converge. Based on non-inferiority margins representing a $10 \%$ and $20 \%$ reduction in event rate, FF/VI 92/22 mcg demonstrated non-inferiority to FP/SAL $250 / 50 \mathrm{mcg}$ with $74 \%$ and $78 \%$ probability, respectively. On the same margins, noninferiority to BUD/FORM 320/9 mcg was demonstrated with $82 \%$ and $86 \%$ probability, respectively (Table 2). However, credible intervals for the comparisons were very wide owing to the weakness of the treatment network. No data were available for the assessment of non-inferiority of FF/VI 184/22 mcg to relevant comparators.

\section{Change from baseline in AQLQ total score}

All ICS/LABA combination therapies included in the model were associated with estimated mean improvements from baseline in AQLQ Total score. However, because of the limited data available for the AQLQ analysis, the $\mathrm{CrI}$ for all treatments were wide and did not exclude zero for the comparison of any active treatment with placebo (Fig. 3). Placebo treatment was associated with an estimated mean (SD) improvement in score of $0.233(0.485)$. The greatest mean (SD) improvement from baseline for this outcome of interest, 0.854 (0.299), was observed with FF/VI 184/22 mcg (Additional file 1: e-table 2). Non-inferiority findings could not be reported for the higher strength of FF/VI; although the higher strength was in the network, none of the comparable treatments of interest were present.

Based on a non-inferiority margin of 0.25 , representing half of the MCID [14], FF/VI 92/22 mcg demonstrated non-inferiority to corresponding doses of FP/SAL 250/50 mcg and BUD/FORM 320/9 mcg with >99 \% and $90 \%$ probability, respectively (Table 2 ). For the non-inferiority margin of 0.5, FF/VI 92/22 mcg demonstrated $>99 \%$ and $96 \%$ probability of non-inferiority to corresponding doses of FP/SAL 250/50 mcg and BUD/FORM 320/9 mcg, respectively. None of the model covariates were found to have a statistically significant effect on outcomes (Additional file 1: e-table 3).

\section{ICS-only subset analysis (Table 3 and Additional file 1: e-table 4)}

In the ICS-only subset analysis of the $\mathrm{FEV}_{1}$ data, the exclusion of two studies permitting LABA use at baseline resulted in a markedly greater observed decrease from baseline $\mathrm{FEV}_{1}$ among patients receiving placebo. For the non-inferiority margin of $75 \mathrm{ml}$, the probabilities 

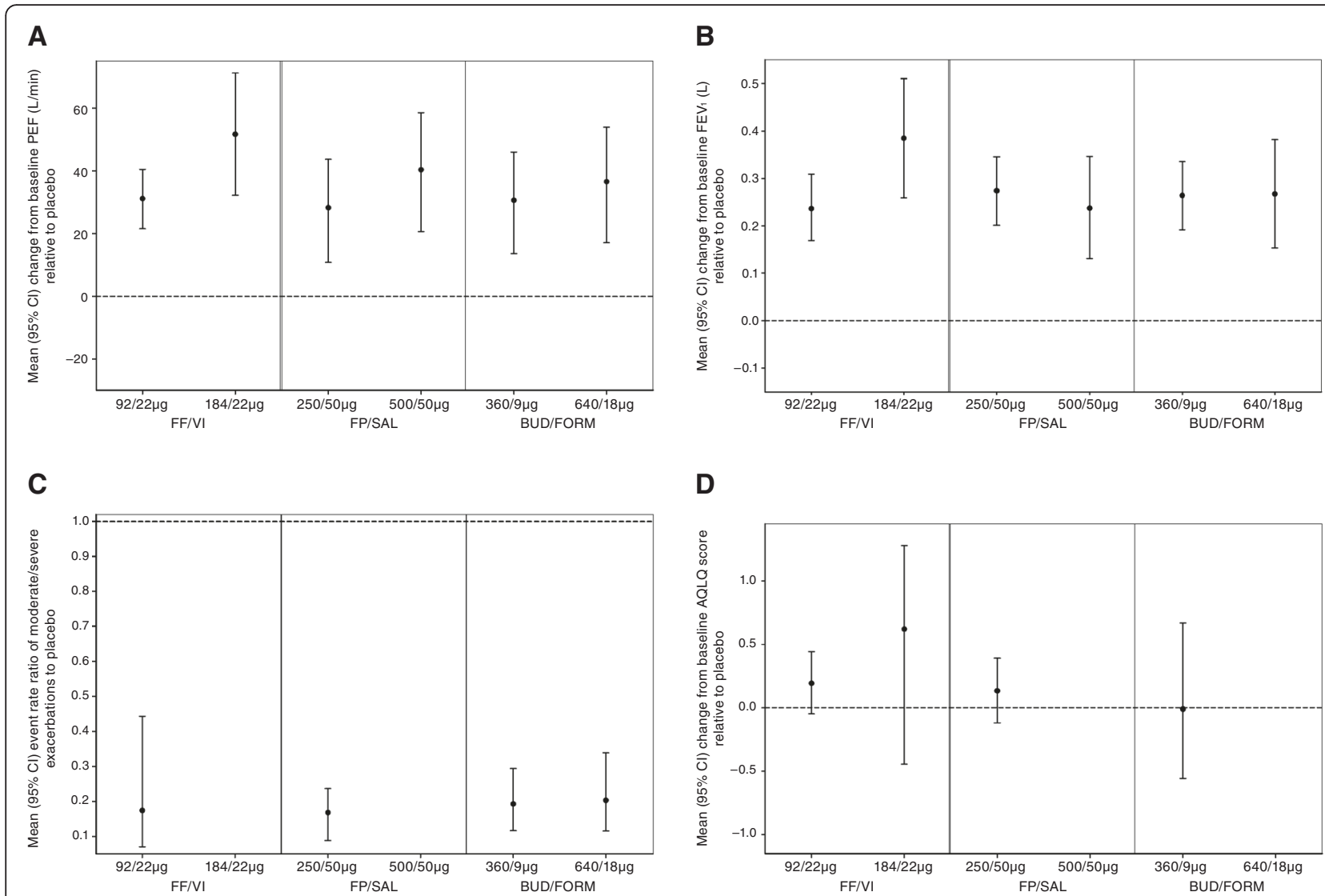

D

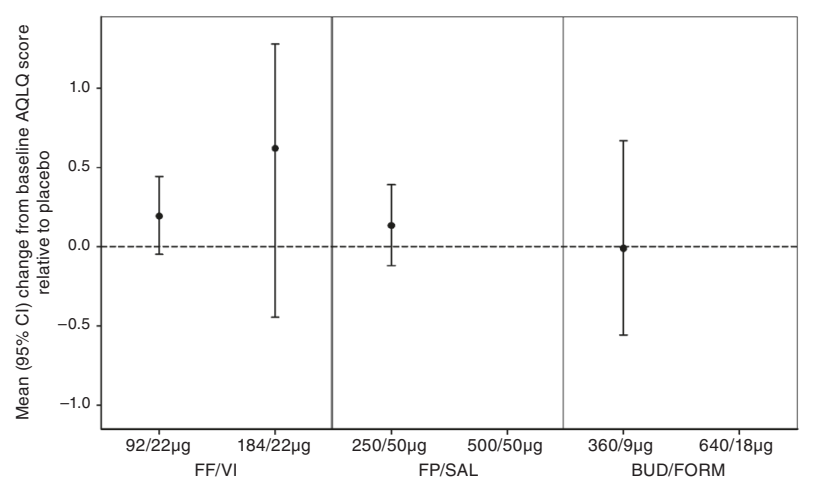

Fig. 2 Change from baseline versus placebo for selected treatments. For studies requiring patients to be treated with ICS or ICS/LABA at baseline; full covariate analysis. a change from baseline in morning PEF. $\mathbf{b}$ change from baseline in $\mathrm{FEV}_{1}$. $\mathbf{c}$ annual rate of moderate/severe exacerbations. d AQLQ Total score. AQLQ = Asthma Quality of Life Questionnaire, BUD = budesonide, FORM = formoterol, FEV ${ }_{1}=$ forced expiratory volume in $1 \mathrm{~s}$, $\mathrm{FF}=$ fluticasone furoate, $\mathrm{FP}=$ fluticasone propionate, $\mathrm{PEF}=$ peak expiratory flow, $\mathrm{SAL}=$ salmeterol, $\mathrm{VI}=$ vilanterol

of non-inferiority were reduced to $84 \%$ and $86 \%$ for comparisons of FF/VI 92/22 mcg with FP/SAL 250/50 mcg and BUD/FORM 400/12 mcg, respectively. The other probabilities of non-inferiority all remained higher than $96 \%$. In the PEF and exacerbations analyses, no studies were excluded in the ICS-only subset analysis for annual moderate/severe exacerbation rate, so the outcomes were unchanged from those of the main analysis.

The ICS-only subset analysis of the AQLQ data excluded one of the seven studies that were included in the main analysis. As a consequence of non-convergence owing to the weakness of the network of six studies, the full covariate model could not be fit. A model including only the study duration covariate was fit. The resulting probability of non-inferiority of FF/VI 92/22 mcg to BUD/ FORM $320 / 9 \mathrm{mcg}$, on a non-inferiority margin of 0.25 , was $93 \%$ and the probability of non-inferiority to FP/SAL $250 / 50 \mathrm{mcg}$ of $>99 \%$ was unchanged. The mean (SD) improvement observed with placebo, 0.177 (0.414), was lower than in the main analysis. The $\mathrm{CrI}$ for the treatment difference vs placebo on the AQLQ outcome excluded zero for both strengths of FF/VI and also for high-dose FP and FF monotherapy; for the remaining treatments in the network, all other comparisons vs placebo included zero.

\section{Sensitivity analysis (Additional file 1: e-table 5)}

Sensitivity analyses, in which additional studies that did not fulfil the primary analysis inclusion criteria were added to the network, were conducted for the PEF and exacerbations outcomes. For PEF, the addition of nine studies which did not report change from baseline over the study duration up to study end to the 18 included in the primary analysis resulted in reductions in the reported probabilities of non-inferiority of FF/VI 92/22 mcg vs FP/SAL 250/50 mcg and BUD/FORM 320/9 mcg, whereas the results for comparisons involving $184 / 22 \mathrm{mcg}$ were similar to those of the primary analysis. For exacerbations, the addition of six studies that withdrew subjects and discontinued followup after an exacerbation to the six included in the primary analysis resulted in a narrowing of the credible intervals for comparisons of FF/VI 92/22 mcg and increased probabilities of non-inferiority vs FP/SAL 250/50 mcg and BUD/FORM $320 / 9 \mathrm{mcg}$. It was also possible, using the sensitivity analysis network, to report a probability of non- 
Table 2 Posterior probability of non-inferiority for FFNI versus other relevant ICS/LABA combination therapies*

\begin{tabular}{|c|c|c|c|c|c|}
\hline \multicolumn{6}{|l|}{ A } \\
\hline \multirow[t]{2}{*}{ Treatment (mcg) } & \multirow[t]{2}{*}{ Comparator (mcg) } & \multirow[t]{2}{*}{ Mean difference, I (95 \% Crl) } & \multicolumn{3}{|c|}{$\begin{array}{l}\text { Probability of non-inferiority } \\
\text { Margin (I/min) }\end{array}$} \\
\hline & & & \multicolumn{2}{|l|}{12} & 15 \\
\hline FFNI 92/22 & FP/SAL 250/50 & $2.832(-12.867-18.531)$ & \multicolumn{2}{|l|}{$97 \%$} & $99 \%$ \\
\hline FFNI 92/22 & BUD/FORM 320/9 & $0.579(-15.155-16.312)$ & \multicolumn{2}{|l|}{$94 \%$} & $98 \%$ \\
\hline FF/NI 184/22 & FP/SAL 500/50 & $11.323(0.289-22.357)$ & \multicolumn{2}{|l|}{$>99 \%$} & $>99 \%$ \\
\hline FF/NI 184/22 & BUD/FORM 640/18 & $15.136(-0.943-31.215)$ & \multicolumn{2}{|l|}{$>99 \%$} & $>99 \%$ \\
\hline \multicolumn{6}{|l|}{ B } \\
\hline \multirow[t]{2}{*}{ Treatment } & \multirow[t]{2}{*}{ Comparator } & \multirow[t]{2}{*}{ Mean difference, ml (95\% Crl) } & \multicolumn{3}{|c|}{$\begin{array}{l}\text { Probability of non-inferiority } \\
\text { Margin (ml) }\end{array}$} \\
\hline & & & 75 & 100 & 125 \\
\hline FF/VI 92/22 & FP/SAL 250/50 & $-36(-92-19)$ & $92 \%$ & $99 \%$ & $>99 \%$ \\
\hline FF/VI 92/22 & BUD/FORM 320/9 & $-27(-98-45)$ & $91 \%$ & $98 \%$ & $>99 \%$ \\
\hline FFNI 184/22 & FP/SAL 500/50 & $147(48-247)$ & $>99 \%$ & $>99 \%$ & $>99 \%$ \\
\hline FF/NI 184/22 & BUD/FORM 640/18 & $118(-19-255)$ & $>99 \%$ & $>99 \%$ & $>99 \%$ \\
\hline \multicolumn{6}{|l|}{$C$} \\
\hline \multirow[t]{2}{*}{ Treatment } & Comparator & Rate ratio $(95 \% \mathrm{Crl})$ & \multicolumn{3}{|c|}{$\begin{array}{l}\text { Probability of non-inferiority } \\
\text { Margin (event rate ratio) }\end{array}$} \\
\hline & & & $10 \%$ & & $20 \%$ \\
\hline FF/VI 92/22 & FP/SAL 250/50 & $1.164(0.428-3.333)$ & $74 \%$ & & $78 \%$ \\
\hline FFNI 92/22 & BUD/FORM 320/9 & $0.985(0.336-2.574)$ & $82 \%$ & & $86 \%$ \\
\hline \multicolumn{6}{|l|}{ D } \\
\hline \multirow[t]{2}{*}{ Treatment } & Comparator & Mean difference, units (95\% Crl) & \multicolumn{3}{|c|}{$\begin{array}{l}\text { Probability of non-inferiority } \\
\text { Margin (units) }\end{array}$} \\
\hline & & & 0.25 & & 0.5 \\
\hline FFNI 92/22 & FP/SAL 250/50 & $0.060(-0.104-0.224)$ & $>99 \%$ & & $>99 \%$ \\
\hline FF/VI 92/22 & BUD/FORM 320/9 & $0.203(-0.461-0.867)$ & $90 \%$ & & $96 \%$ \\
\hline
\end{tabular}

For studies requiring patients to be treated with ICS or ICS/LABA at baseline; full covariate model

*Other relevant ICS/LABA: FP/SAL 250/50 mcg and 500/50 mcg; BUD/FORM 320/9 mcg and 640/18 mcg

a) change from baseline in morning PEF. b) change from baseline in $\mathrm{FEV}_{1}$. c) annual rate of moderate/severe exacerbations ${ }^{\dagger}$. d) AQLQ Total score

${ }^{\dagger}$ Only study length was included as a covariate in analysis of moderate/severe exacerbations data

Note: All stated doses are mcg

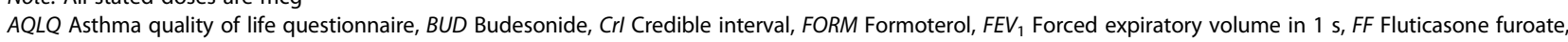

FP Fluticasone propionate, PEF Peak expiratory flow, SAL Salmeterol, VI Vilanterol

inferiority for FF/VI 184/22 mcg vs BUD/FORM 640/18 mcg on exacerbations; however, the CrI for this estimate is wide. Thus, the sensitivity analysis demonstrates that the results for the exacerbations network are susceptible to the studies that are included in the network. The findings of a separate sensitivity analysis in which the exacerbations analysis was rerun with alternate person-years definitions were similar to those of the primary analysis.

\section{Assessment of alternative modelling approaches (Additional file 1: e-table 6)}

The findings and details of the post-hoc analysis utilising alternative modelling approaches-specifically, a frequentist analysis using a random effects model with fixed study and treatment effects, and a pairwise contrast analysis ( $\mathrm{FEV}_{1}$ endpoint only)-are reported in Additional file 1: e-table 6. The results of these analyses showed that, where the application of the varied methodologies to the dataset was feasible, PEF, FEV 1 and AQLQ results using these methodologies were consistent with those of the primary MTC analyses.

\section{Discussion}

FF/VI represents the first once-daily ICS/LABA combination to be approved for use in the treatment of asthma; two strengths of FF/VI have been approved in Europe and Japan. We sought to compare the clinical efficacy of FF/VI 92/22 mcg with that of twice-daily FP/SAL 250/50 $\mathrm{mcg}$ and BUD/FORM 320/9 mcg, and that of FF/VI 184/22 mcg with FP/SAL 500/50 mcg and BUD/FORM $640 / 18 \mathrm{mcg}$. FF/VI $92 / 22 \mathrm{mcg}$ has previously been shown to be comparable in efficacy in improving lung 
Table 3 Posterior probability of non-inferiority for FF/VI versus other relevant ICS/LABA combination therapies*

\begin{tabular}{|c|c|c|c|c|c|}
\hline \multicolumn{6}{|l|}{ A } \\
\hline \multirow[t]{2}{*}{ Treatment } & \multirow[t]{2}{*}{ Comparator } & \multirow[t]{2}{*}{$\begin{array}{l}\text { Mean difference, } \mathrm{ml} \\
(95 \% \mathrm{Crl})\end{array}$} & \multicolumn{3}{|c|}{$\begin{array}{l}\text { Probability of non-inferiority } \\
\text { Margin (ml) }\end{array}$} \\
\hline & & & 75 & 100 & 125 \\
\hline FF/NI 92/22 & FP/SAL 250/50 & $-0.046(-0.102-0.010)$ & $84 \%$ & $97 \%$ & $>99 \%$ \\
\hline FFNI 92/22 & BUD/FORM 320/9 & $-0.037(-0.106-0.032)$ & $86 \%$ & $96 \%$ & $99 \%$ \\
\hline FFNI 184/22 & FP/SAL 500/50 & $0.163(0.058-0.269)$ & $>99 \%$ & $>99 \%$ & $>99 \%$ \\
\hline FFNI 184/22 & BUD/FORM 640/18 & $0.099(-0.043-0.241)$ & $99 \%$ & $>99 \%$ & $>99 \%$ \\
\hline \multicolumn{6}{|l|}{ B } \\
\hline \multirow[t]{2}{*}{ Treatment } & Comparator & $\begin{array}{l}\text { Mean difference, units } \\
(95 \% \mathrm{Crl})\end{array}$ & \multicolumn{3}{|c|}{$\begin{array}{l}\text { Probability of non-inferiority } \\
\text { Margin (units) }\end{array}$} \\
\hline & & & 0.25 & & 0.5 \\
\hline FF/NI 92/22 & FP/SAL 250/50 & $93(-43-230)$ & $>99 \%$ & & $>99 \%$ \\
\hline FF/NI 92/22 & BUD/FORM 320/9 & $89(-502-680)$ & $93 \%$ & & $95 \%$ \\
\hline
\end{tabular}

function and health status with FP/SAL 250/50 mcg in a head-to-head randomised controlled trial [7].

Using an MTC approach, we examined the probability of non-inferiority of once-daily FF/VI compared with corresponding strengths of twice-daily FP/SAL and BUD/FORM by combining data on clinical efficacy outcomes from several RCTs. All three ICS/LABA combination therapies have previously been shown to be associated with improvements in these outcomes relative to placebo.

We chose to use a Bayesian, hierarchical MTC modelling approach to synthesise evidence from RCTs conducted in adolescents and adults with asthma that involved at least one ICS/LABA comparator. Broad-scope searches were used to identify as many studies as possible that were potentially suitable for inclusion in the MTC. Exclusion criteria were subsequently applied on an outcome-byoutcome basis. The Bayesian approach that we used enabled a probabilistic estimate of non-inferiority to be generated directly from the posterior distribution [22].

To output meaningful probabilities of non-inferiority, it was necessary to select suitable margins. The noninferiority margins used for the PEF analysis in this study, $12 \mathrm{l} / \mathrm{min}$ and $15 \mathrm{l} / \mathrm{min}$, are well-established margins used in numerous previous studies and based upon European Medicines Agency guidelines [11, 23, 24]. For $\mathrm{FEV}_{1}$, the margins of $75 \mathrm{ml}, 100 \mathrm{ml}$ and $125 \mathrm{ml}$ were chosen based upon the minimal clinically important difference (MCID) of $230 \mathrm{ml}$ [13] and non-inferiority margins used in previous comparative studies involving FF/VI [6] or FF [25]. For exacerbations, the non-inferiority margins of $10 \%$ and $20 \%$ rate ratio reductions are consistent with the margin of 1.18 used in a previous non-inferiority study of FP/SAL vs FP [12]. The accepted MCID for the AQLQ is 0.5 [14], hence the use of a margin of 0.25 , representing half of the MCID, preserves $50 \%$ of the active comparator effect. We consider that, as these margins are smaller than the MCID and therefore imply higher thresholds for demonstrating non-inferiority between treatments, they represent conservative margins for the non-inferiority analysis. However, it is important to note that a finding of a low probability of non-inferiority, using such conservative margins, does not imply lack of comparability or inferiority.

Based on these conservative margins, the results of the primary MTC analyses suggest that there is a high probability that FF/VI $92 / 22 \mathrm{mcg}$ is non-inferior to $\mathrm{FP} / \mathrm{SAL}$ 250/50 mcg and BUD/FORM 320/9 mcg on lung function (PEF and $\mathrm{FEV}_{1}$ ) and health status (AQLQ) endpoints, supporting the findings of the previous head-to-head RCT of FF/VI 92/22 mcg compared with FP/SAL 250/50 mcg. The analysis of exacerbation rate was inconclusive owing to the lack of sufficient data and disconnectedness of the network.

The effectiveness of any meta-analytic method can be limited by the amount of clinical trial data available for each of the treatments in the analysis. As a consequence of the limited number of RCTs available to inform comparisons of ICS/LABA therapies in asthma, the CrI for most of the non-inferiority estimates in our study were wide. Moreover, we decided a priori to treat different strengths of the same therapies as different treatments. This represents both a strength-in that the comparisons we have studied are more precise-and a weakness of our approach, as combining dosages may have produced a stronger and more 
connected network for the lung function and health status outcomes. The CrI were particularly wide for the outcomes of secondary interest (i.e. exacerbations and AQLQ). Exacerbations, in particular, are relatively rare events and are therefore typically only examined in longer-term studies. In addition, the primary endpoints of most RCTs in asthma assess lung function, rather than exacerbations. As such, our study network was weak with respect to exacerbations; this MTC incorporated only six studies, including just one study of FF/VI 92/22 mcg [5] and no studies of FF/VI 184/ $22 \mathrm{mcg}$ for the primary exacerbations analysis. The small number of studies led to limited information in the network and subsequent disconnections in treatment networks. The full model did not converge because of the weak network. One consequence of this was that we were unable to account for covariates in the exacerbations MTC as was possible for the other three MTC outcomes. The post-hoc assessment of alternative modelling approaches showed that the findings of the lung function and health status MTCs were consistent upon the application of varied methodologies, including a frequentist approach, to the data where the network was sufficiently connected to permit this.

Population and endpoint heterogeneity in the studies included in the MTC analyses was addressed as far as possible by our inclusion criteria to ensure that patient populations were suitable for comparison. To minimise study-to-study variability, studies were only included in the primary PEF analysis if they reported mean change from baseline averaged across the whole trial, rather than from baseline to a specific timepoint. For the same reason, studies were excluded from the exacerbations analysis if the definition of exacerbation differed considerably from that set out in the ATS/ERS Task Force recommendation [8]. The findings of sensitivity analyses of both of these outcomes, conducted using data from enlarged networks derived from relaxed inclusion criteria, suggest that the MTC findings are highly susceptible to the addition of studies to the network.

Despite the measures we took in order to reduce study-to-study variability as far as possible given the nature of the analysis, over-dispersion was observed in the primary analysis model distributions. Through study-level covariate modelling within the MTC, we assessed whether variables including study duration, average patient age and exacerbation history affected the comparisons; we sought to correct for this through the incorporation of heterogeneity factors at the study level. Significant covariate effects of baseline $\mathrm{FEV}_{1}$ and study duration were observed in the PEF analysis only. The observation that greater baseline $\mathrm{FEV}_{1}$ is associated with slightly greater improvement from baseline in PEF is consistent with incompleteness of reversibility in patients with more severe airflow limitation [26].
Still, a limitation of this approach that should be borne in mind in the interpretation of the results is that it is not possible to correct for all confounding factors, particularly as patient-level data were not available. For instance, although the study inclusion criteria specified that patients had to be uncontrolled/symptomatic at baseline, it was not possible to ensure a consistent definition of "uncontrolled" across studies as the required data was often not reported. Furthermore, the fact that we have synthesised evidence from trials conducted at different periods of time and in different regions means that there will inevitably be some degree of underlying variation that could have influenced the findings. For instance, basic standards of care vary across regions and typically improve over time.

Although MTC are established as a useful tool for the synthesis of evidence that is suitable for use in clinical decision-making [27], to our knowledge, this is the first study to apply this methodology to data from clinical trials in asthma to investigate the relative efficacy of specific treatments. In a recently-published network meta-analysis in which data on treatment interventions in asthma were combined across classes [28] and their effectiveness assessed, ICS/LABA combination therapies were found to be the most effective intervention for the prevention of asthma exacerbations. In COPD, findings of meta-analyses of treatment efficacy data $[29,30]$ have indicated that ICS/ LABA combination therapy have a greater positive effect on COPD outcomes than alternative treatment modalities.

\section{Conclusions}

The findings of the MTC suggest that the efficacy of both strengths of once-daily FF/VI in asthma is broadly comparable to that of corresponding doses of established twicedaily ICS/LABA combinations, FP/SAL and BUD/FORM, on lung function and health status outcomes of interest in the primary study populations. The MTC supported the findings of a previously-reported head-to-head randomised controlled trial of FF/VI 92/22 mcg vs FP/SAL 250/50 mcg in which it was shown that the efficacy of these treatments in improving lung function and health status endpoints is similar [7]. It should be borne in mind that the MTC findings are obtained through the analysis of outcomes from RCTs and any potential efficacy benefits that may derive from treatment attributes such as once- vs twice-daily dosing in real-world clinical practice will not be reflected in these data.

\section{Supplementary data update}

The mixed treatment comparison was updated with more recently published data; studies published between 18th December 2012 and 30th July 2014 were identified by an update of the systematic review. Seven additional studies were identified for inclusion in this update, thus a total of 38 studies were included and are presented in 
the study supplement (Additional file 2: Tables S1-S3). The results including these data are in line with those of the original analyses, and thus did not affect any of the conclusions previously drawn.

\section{Additional files}

Additional file 1: e-table 1: Characteristics of the studies and treatment arms included in the MTC. e-table 2: Results of mixed treatment comparisons for ICS/LABA treatments of interest (full covariate model, if available). e-table 3: Summary of findings of covariate analysis, by outcome of interest (primary analysis population; full covariate model, if available). e-table 4: Results of mixed treatment comparisons by ICS/LABA treatment. e-table 5: Posterior probability of non-inferiority for FF/NI versus other relevant ICS/LABA combination therapies*, sensitivity analysis. e-table 6: Outcomes of assessment of alternative modelling approaches. Supplementary figure 1: Networks of study treatments, by outcome of interest (sensitivity analysis population). (PDF $705 \mathrm{~kb}$ )

Additional file 2: Table S1: Summary of studies and treatment arms included in the updated primary mixed treatment comparison analysis. Table S2: Posterior probability of non-inferiority for FFNI versus other relevant ICS/LABA combination therapies Table S3: Posterior probability of non-inferiority for FFNI versus other relevant ICS/LABA combination therapies. (DOCX $22 \mathrm{~kb}$ )

\section{Abbreviations}

AQLQ: Asthma quality of life questionnaire; ATS: American thoracic society; BUD: Budesonide; Crl: Credible interval; ERS: European respiratory society; FORM: Formoterol; $\mathrm{FEV}_{1}$ : Forced expiratory volume in $1 \mathrm{~s}$; FF: Fluticasone furoate; FP: Fluticasone propionate; ICS: Inhaled corticosteroid; LABA: Long-acting beta $_{2}$ agonist; MCID: Minimum clinically important difference; MTC: Mixed treatment comparison; PEF: Peak expiratory flow:

RCT: Randomised controlled trial; SAL: Salmeterol; SD: Standard deviation; VI: Vilanterol.

\section{Competing interests}

$H S, G S, L F$, and DL are employees of and hold stock in GSK. JW and EC were employees of GSK at the time of the study. SB and MD were paid a consulting fee for conducting meta-analytic analyses, but were not paid to write the article and declare no other competing interests.

\section{Authors' contributions}

$\mathrm{HS}, \mathrm{SB}$ and MD contributed to the conception and design of the study and the analysis and interpretation of the data. GS contributed to the conception and design of the study, the acquisition of the data, and the interpretation of the data. JW and EC contributed to the conception and design of the study, the acquisition of the data, and data analysis and interpretation. LF and DL contributed to the conception and design of the study and the interpretation of data. All authors read and approved the final manuscript.

\section{Authors' information}

Value Evidence and Outcomes, GlaxoSmithKline, Stockley Park West, Blg 10, 1-3 Iron Bridge Road, Uxbridge, Middlesex UB11 1BT, UK is the former affiliation for JW.

\section{Acknowledgements}

We wish to acknowledge the contribution of Helen Birch and Nicholas Galwey (GlaxoSmithKline) to the statistical analysis. Editorial support in the form of development of the draft outline and manuscript first draft in consultation with the authors, editorial suggestions to draft versions of this paper, assembling tables and figures, collating author comments, copyediting, fact checking, referencing and graphic services was provided by Ian Grieve, PhD at Gardiner-Caldwell Communications (Macclesfield, UK) and was funded by GSK.

\section{Funding}

The study was funded by GlaxoSmithKline (GSK study number: HO-12-10140). Employees of the sponsor participated in the conception and design of the study and interpretation of the data.

\section{Author details}

'Value Evidence and Outcomes, GlaxoSmithKline, Stockley Park West, Blg 10, 1-3 Iron Bridge Road, Uxbridge, Middlesex UB11 1BT, UK. ${ }^{2}$ Respiratory Medicines Development Centre, GlaxoSmithKline, Stockley Park, UK. ${ }^{3}$ Health Investment Evidence (Formerly), Global Health Outcomes, GlaxoSmithKline, Stockley Park, UK. ${ }^{4}$ Berry Consultants LLC, Austin, TX, USA.

Received: 11 December 2014 Accepted: 16 December 2015 Published online: 08 February 2016

\section{References}

1. Rossios C, To Y, To M, Ito M, Kharitonov SA, Barnes PJ, et al. Long-acting fluticasone furoate has a superior pharmacological profile to fluticasone propionate in human respiratory cells. Eur J Pharmacol. 2011;670:244-51.

2. Slack RJ, Barrett VJ, Morrison VS, Sturton RG, Emmons AJ, Ford AJ, et al. In vitro pharmacological characterization of vilanterol, a novel long acting B2-adrenoceptor agonist with 24 hour duration of action. J Pharmacol Exp Ther. 2013;344:218-30.

3. Bleecker ER, Lötvall J, O'Byrne PM, Woodcock A, Busse WW, Kerwin EM, et al. Fluticasone furoate/vilanterol $100 / 25 \mathrm{mcg}$ compared with fluticasone furoate 100mcg in asthma: a randomized trial. J Allergy Clin Immunol Pract. 2014;2: 553-61.

4. Busse WW, O'Byrne PM, Bleecker ER, Lötvall J, Woodcock A, Andersen L, et al. Safety and tolerability of the novel inhaled corticosteroid fluticasone furoate in combination with the $\beta 2$ agonist vilanterol administered once daily for 52 weeks in patients $\geq 12$ years old with asthma: a randomised trial. Thorax. 2013;68:513-20.

5. Bateman ED, O'Byrne PM, Busse WW, Lötvall J, Bleecker ER, Andersen L, et al. Once-daily fluticasone furoate (FF)/vilanterol reduces risk of severe exacerbations in asthma versus FF alone. Thorax. 2014;69:312-9.

6. O'Byrne PM, Bleecker ER, Bateman ED, Busse WW, Woodcock A, Forth R, et al. Once-daily fluticasone furoate alone or combined with vilanterol in persistent asthma. Eur Respir J. 2014;43:773-82.

7. Woodcock A, Bleecker ER, Lötvall J, O'Byrne PM, Bateman ED, Medley H, et al. Efficacy and safety of fluticasone furoate/vilanterol compared with fluticasone propionate/salmeterol combination in adult and adolescent patients with persistent asthma: a randomized trial. Chest. 2013;144:1222-9.

8. Reddel HK, Taylor DR, Bateman ED, Boulet LP, Boushey HA, Busse WW, et al. An official American Thoracic Society/European Respiratory Society statement: asthma control and exacerbations: standardizing endpoints for clinical asthma trials and clinical practice. Am J Respir Crit Care Med. 2009;180:59-99.

9. Shingo S, Zhang J, Reiss TF. Correlation of airway obstruction and patientreported endpoints in clinical studies. Eur Respir J. 2001:17:220-4.

10. O'Byrne PM, Barnes PJ, Rodriguez-Roisin R, Runnerstrom E, Sandstrom T, Svensson $\mathrm{K}$, et al. Low-dose inhaled budesonide and formoterol in mild persistent asthma: the OPTIMA randomized trial. Am J Respir Crit Care Med. 2001:164:1392-7.

11. Godard P, Greillier P, Pigearias B, Nachbaur G, Desfougeres J-L, Attali V. Maintaining asthma control in persistent asthma: Comparison of three strategies in a 6-month double-blind randomised study. Respir Med. 2008:102:1124-31.

12. Chuchalin A, Jacques L, Frith L. Salmeterol/fluticasone propionate via Diskus $^{\text {TM }}$ once daily versus fluticasone propionate twice daily in patients with mild asthma not previously receiving maintenance corticosteroids. Clin Drug Invest. 2008:3:169-81.

13. Santanello NC, Zhang J, Seidenberg B, Reiss TF, Barber BL. What are minimal important changes for asthma measures in a clinical trial? Eur Respir J. 1999; 14:23-7.

14. Juniper EF, Guyatt GH, Willan A, Griffith LE. Determining a minimal important change in a disease-specific quality of life questionnaire. J Clin Epidemiol. 1994;47:81-7.

15. Smith TC, Spiegelhalter DJ, Thomas A. Bayesian approaches to randomeffects meta-analysis: a comparative study. Stat Med. 1995;14:2685-99.

16. Sutton AJ, Abrams KR. Bayesian methods in meta-analysis and evidence synthesis. Stat Methods Med Res. 2001;10:277-303.

17. Sutton AJ, Higgins JP. Recent developments in meta-analysis. Stat Med. 2008;27:625-50 
18. Berry SM, Shane Reese C, Larkey PD. Bridging different eras in sports. J Amer Stat Assoc. 1999;94:661-76.

19. Gilks WR, Richardson S. Spiegelhalter D. Markov Chain Monte Carlo In Practice: Chapman \& Hall; 1996.

20. van Valkenhoef G, Lu G, de Brock B, Hillege H, Ades AE, Welton NJ. Automating network meta-analysis. Res Synth Methods. 2012;3:285-99.

21. Lunn DJ, Thomas A, Best N, Spiegelhalter D. WinBUGS - a Bayesian modelling framework: concepts, structure and extensibility. Statistics and Computing. 2000;10:325-37.

22. Gelman A, Carlin JB, Stern HS. Rubin DB. Second Edition. Chapman \& Hall/ CRC: Bayesian Data Analysis; 2003

23. Bateman ED, Silins V, Bogolubov M. Clinical equivalence of salmeterol/fluticasone propionate in combination (50/100 $\mu \mathrm{g}$ twice daily) when administered via a chlorofluorocarbon-free metered dose inhaler or dry powder inhaler to patients with mild-to-moderate asthma. Respir Med. 2001:85:136-46.

24. Van Noord JA, Lill H, Carrillo Diaz T, Greefhorst AP, Davies P. Clinical equivalence of a salmeterolfluticasone propionate combination product (50/500 $\mu \mathrm{gg})$ delivered via a chlorocarbon-free metered dose inhaler with the Diskus ${ }^{T \mathrm{~T}}$ in patients with moderate to severe asthma. Clin Drug Invest. 2001;21:243-55.

25. Woodcock A, Bleecker ER, Busse WW, Lötvall J, Snowise NG, Frith L, et al. Fluticasone furoate: once-daily evening treatment versus twice-daily treatment in moderate asthma. Respir Res. 2011:12:160

26. Brown PJ, Greville HW, Finucane KE. Asthma and irreversible airflow obstruction. Thorax. 1984;39:131-6.

27. Caldwell DM, Ades AE, Higgins JP. Simultaneous comparison of multiple treatments: combining direct and indirect evidence. BMJ. 2005:331:897-900.

28. Loymans RJ, Gemperli A, Cohen J, Rubinstein SM, Sterk PJ, Reddel HK, et al. Comparative effectiveness of long term drug treatment strategies to prevent asthma exacerbations: network meta-analysis. BMJ. 2014;348:93009.

29. Baker WL, Baker EL, Coleman Cl. Pharmacologic treatments for chronic obstructive pulmonary disease: a mixed-treatment comparison metaanalysis. Pharmacotherapy. 2009;29:891-905.

30. Mills EJ, Druyts E, Ghement I, Puhan MA. Pharmacotherapies for chronic obstructive pulmonary disease: a multiple treatment comparison metaanalysis. Clin Epidemiol. 2011;3:107-29.

\section{Submit your next manuscript to BioMed Central} and we will help you at every step:

- We accept pre-submission inquiries

- Our selector tool helps you to find the most relevant journal

- We provide round the clock customer support

- Convenient online submission

- Thorough peer review

- Inclusion in PubMed and all major indexing services

- Maximum visibility for your research

Submit your manuscript at www biomedcentral.com/submit

\section{(O) BioMed Central}

